\title{
Resonant tunnelling between the chiral Landau states of twisted graphene lattices
}

\author{
M.T. Greenaway ${ }^{1}$, E.E. Vdovin ${ }^{1,2,3}$, A. Mishchenko ${ }^{4}$, O. Makarovsky ${ }^{1}$, A. Patanè ${ }^{1}$, J.R. Wallbank ${ }^{5}$, \\ Y. $\mathrm{Cao}^{6}$, A.V. Kretinin ${ }^{6}$, M.J. Zhu ${ }^{4}$, S. V. Morozov ${ }^{2,3}$, \\ V.I. Fal'ko ${ }^{5}$, K.S. Novoselov ${ }^{4}$, A.K. Geim ${ }^{4,6}$, T.M. Fromhold ${ }^{1}$ and L. Eaves ${ }^{1,4}$ \\ ${ }^{1}$ School of Physics and Astronomy, University of Nottingham NG7 2RD UK \\ ${ }^{2}$ Institute of Microelectronics Technology and High Purity Materials, RAS, Chernogolovka 142432, Russia \\ ${ }^{3}$ National University of Science and Technology "MISiS", 119049, Leninsky pr.4, Moscow, Russia \\ ${ }^{4}$ School of Physics and Astronomy, University of Manchester, M13 9PL, UK \\ ${ }^{5}$ Physics Department, Lancaster University, Lancaster LA1 4YB, UK \\ ${ }^{6}$ Centre for Mesoscience and Nanotechnology, University of Manchester, M13 9PL, UK
}

(Dated: July 14, 2016)

Paper published on 19th October 2015: Nature Physics 11, 10571062 (2015), doi:10.1038/nphys 3507

A class of multilayered functional materials has recently emerged in which the component atomic layers are held together by weak van der Waals forces that preserve the structural integrity and physical properties of each layer. An exemplar of such a structure is a transistor device in which relativistic Dirac Fermions can resonantly tunnel through a boron nitride barrier, a few atomic layers thick, sandwiched between two graphene electrodes. An applied magnetic field quantises graphene's gapless conduction and valence band states into discrete Landau levels, allowing us to resolve individual inter-Landau level transitions and thereby demonstrate that the energy, momentum and chiral properties of the electrons are conserved in the tunnelling process. We also demonstrate that the change in the semiclassical cyclotron trajectories, following an inter-layer tunnelling event, is analogous to the case of intralayer Klein tunnelling.

An electron moving through the hexagonal crystal structure of graphene is not only quasi-relativistic but also exhibits chirality [1]. The chirality originates from the diatomic unit cell of the graphene crystal lattice and means that the amplitude of the electron wavefunction is intrinsically coupled to the direction of motion. This gives rise to the phenomenon of Klein tunnelling whereby an electron can pass with unity transmission through a potential barrier formed in the graphene layer $[2,3]$. In principle, chirality should also affect the properties of devices in which electrons tunnel between graphene layers. To investigate this effect, we focus on a van der Waals heterostructure [4] in which Dirac fermions can resonantly tunnel between two graphene electrodes separated by a hexagonal boron nitride tunnel barrier [5-8]. Recent work has demonstrated that even a small misalignment of the crystalline lattices of the two graphene electrodes lowers the translation symmetry in the plane of the tunnel barrier and gives rise to an impulse which
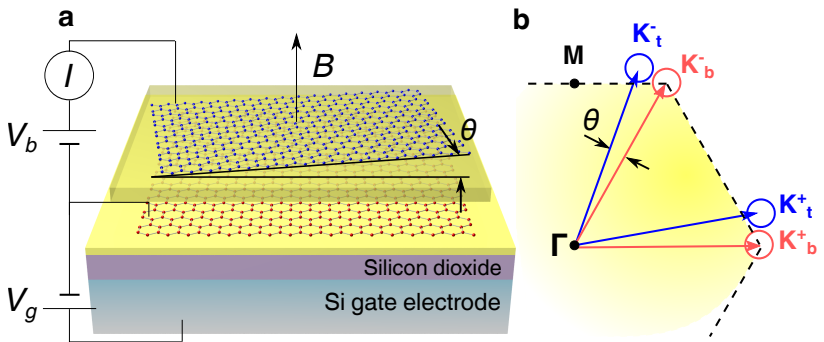

Figure 1: Device structure and misaligned Brillouin zones. a Schematic of the device showing the two misaligned graphene lattices (bottom, red and top, blue) separated by a boron nitride tunnel barrier (upper yellow region). An optical micrograph is shown in Fig. 1, SI. $\mathbf{b}$ dashed black lines show the Brillouin zone boundary for electrons in the bottom graphene layer. Red arrows show the vector positions of the Dirac points $\mathbf{K}_{\mathbf{b}} \pm$ (red circles) relative to the $\Gamma$ point. Blue arrows show the positions of the Dirac points in the top layer, $\mathbf{K}_{\mathbf{t}}^{ \pm}$(blue circles), misoriented at an angle $\theta$ to the bottom layer.

modifies the dynamics of the tunnelling electron [7-12]. By applying a magnetic field perpendicular to the layers we show that electron tunnelling is governed by the laws of conservation of energy and in-plane momentum, as was demonstrated for the case of inter-Landau level tunnelling between two quantum wells in III-V semiconductor heterostructures [13, 14]. Here, our measurements on electron tunnelling between two graphene layers reveal an additional phenomenon namely, chirality, and its enhancement by the quantising magnetic field.

Our device, with bias, $V_{b}$, and gate, $V_{g}$, voltages applied, is shown schematically in Fig 1a. It consists of a 4-layer thick $(d=1.4 \mathrm{~nm})$ hexagonal boron nitride (hBN) tunnel barrier [15] sandwiched between two high purity crystalline graphene electrodes. The active area of the device, in which tunneling occurs, is $100 \mu \mathrm{m}^{2}$. The lower graphene electrode sits on an atomically-flat hBN layer (lower yellow region in Fig 1a), directly above a $\mathrm{SiO}_{2} / \mathrm{n}-\mathrm{Si}$ substrate, in which the doped Si layer acts as the gate electrode and the oxide thickness is $300 \mathrm{~nm}$. Our device is thus a graphene-boron nitride analogue of an independently-contacted GaAs/AlGaAs double quan- 

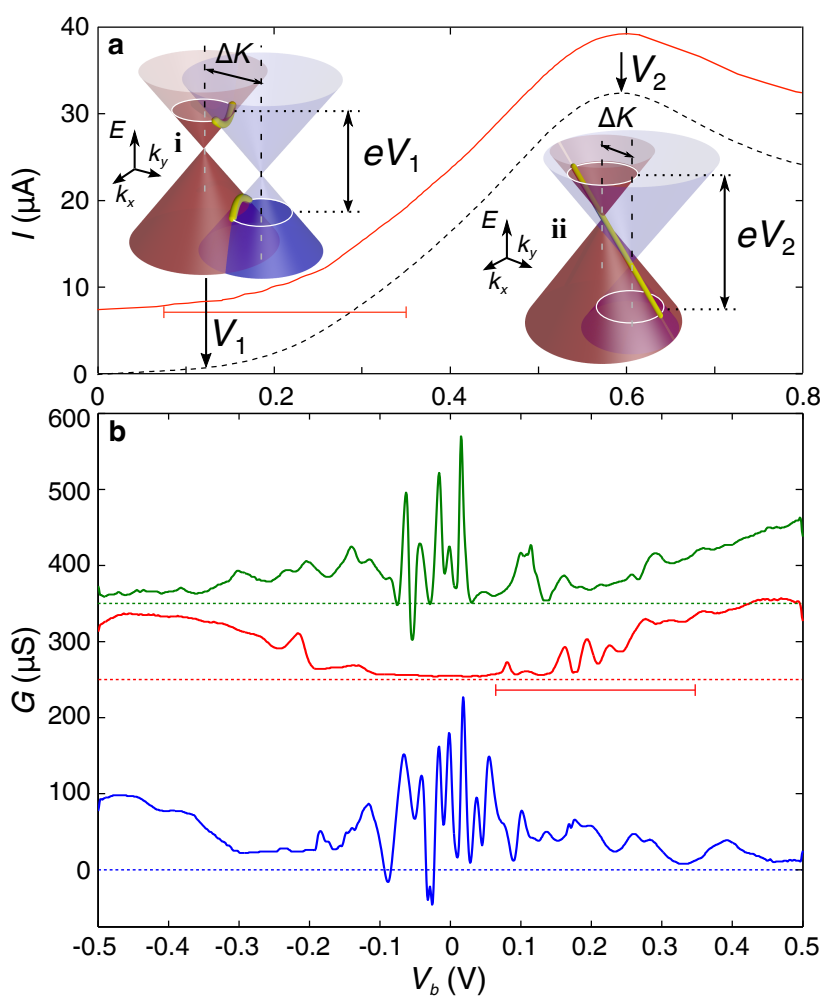

Figure 2: Magnetic field-induced resonances in the conductance. a $I\left(V_{b}\right)$ curves measured when $V_{g}=0$ for $B=0$ (black dashed) and $4 \mathrm{~T}$ (red solid), the latter offset by 7.5 $\mu \mathrm{A}$ for clarity. Insets $\mathbf{i}$ and $\mathbf{i i}$ show the relative energies of the Dirac cones, displaced by $\Delta K$, in the bottom (red) and top (blue) electrodes at the voltages $V_{1}$ and $V_{2}$ marked by the labelled vertical arrows. The intersections of the cones are shown by the thick yellow curves. The Fermi circles of the two layers are shown in white. $\mathbf{b}$ differential conductance, $G\left(V_{b}\right)$, measured at $V_{g}=-40 \mathrm{~V}$ (blue lower curve), $V_{g}=0 \mathrm{~V}$ (red middle curve), and $V_{g}=40 \mathrm{~V}$ (green upper curve) when $B=4 \mathrm{~T}$ and temperature $T=4 \mathrm{~K}$. Red and green curves are offset by $250 \mu \mathrm{S}$ and $350 \mu \mathrm{S}$, respectively i.e. dotted lines mark $G=0$ for the three curves. Red horizontal bars in $\mathbf{a}, \mathbf{b}$ mark the $V_{b}>0$ region where conductance peaks can be observed when $V_{g}=0$.

tum well structure with back gate [13]. The two graphene lattices are intentionally aligned to within an angle of $1^{\circ}$, see ref. [8] for details. However, even this slight misalignment, or "twist angle", $\theta$, leads to a significant $k$-space displacement of magnitude $\Delta K=\left|\Delta \mathbf{K}^{ \pm}\right|=$ $\left|\mathbf{K}_{b}^{ \pm}-\mathbf{K}_{t}^{ \pm}\right|=2 \sin (\theta / 2)\left|\mathbf{K}_{b}^{ \pm}\right|$of the Dirac cones at the Brillouin zone corners [16-19], see Fig. 1b and Fig. 2a, insets. This displacement induces an impulse on a tunnelling electron and has a large effect on the measured current-voltage characteristics and their magnetic field dependence.

Fig. 2a (black dashed curve) shows the measured current-voltage curve, $I\left(V_{b}\right)$, at $V_{g}=0$ in the absence of a magnetic field. The current increases at a threshold bias voltage $V_{1}$ and reaches a resonant peak when $V_{b}=V_{2}=0.58 \mathrm{~V}$, beyond which there is a region of negative differential conductance (NDC). When $V_{b}=V_{1}$, see inset $\mathbf{i}$ of Fig. 2a, the Fermi circle in one cone partially overlaps with empty states in the other, so that electrons can tunnel with energy and momentum conservation $[8,9]$. When $V_{b}=V_{2}$ (inset ii) the cones intersect along a straight line and the current reaches a resonant maximum. The NDC observed for $V_{b}>V_{2}$ occurs when the intersection between the Dirac cones changes from a straight line to an ellipse, thereby reducing the number of states that contribute to the tunnel current (see Supplementary Video).

A magnetic field, $\mathbf{B}$, applied perpendicular to the graphene layers quantises the electron energy into a spectrum of unequally-spaced LLs defined by $E_{n_{b, t}}=$ $\operatorname{sgn}\left(n_{b, t}\right) \sqrt{2\left|n_{b, t}\right|} \hbar v_{F} / l_{B}$, where $n_{b, t}$ is the LL index in the bottom (b) and top (t) layers, and $l_{B}=\sqrt{\hbar / e B}[20-$ 32]. By comparing our measured tunnel current with transfer Hamiltonian calculations, we demonstrate the composite spatial-spinor form of the quantised Landau states and the effect of chirality on the measured currentvoltage characteristics. In addition, by using a semiclassical description of the cyclotron orbits of an electron before and after the tunnelling event, we explain how resonant tunnelling is enabled by the large momentum impulse induced by the small twist angle between the two graphene lattices.

\section{Effect of a perpendicular magnetic field on resonant tunnelling: experiment and theory}

Landau level quantisation induces weak features in $I\left(V_{b}\right)$ when $V_{g}=0$ for $0.08 \mathrm{~V}<V_{b}<0.35 \mathrm{~V}$ (see region of the red curve in Fig. 2a indicated by the red horizontal bar) and sharp, large amplitude, resonant features in the differential conductance, $G\left(V_{b}\right)=d I / d V_{b}$, as shown in Fig. 2b for gate voltages $V_{g}=0$ and $\pm 40 \mathrm{~V}$. By combining similar plots at intermediate gate voltages, we generate the colour maps of $G\left(V_{b}, V_{g}\right)$ shown in Figs. $8 \mathbf{a}$ and c, for $B=2$ and $4 \mathrm{~T}$, respectively. The regions of high conductance are patterned by small "islands" that originate from resonant tunnelling of electrons when LLs in the two graphene layers become aligned in energy (shown schematically in Fig. 4a,b). We observe no evidence of lifting of spin- and valley- degeneracies in the $G\left(V_{b}, V_{g}\right)$ plots at these magnetic fields. These islands are sharply defined for $\left|V_{b}\right| \lesssim 0.2 \mathrm{~V}$ but become broadened at high $\left|V_{b}\right|$, which could arise from carrier heating due to high current levels and/or increased lifetime broadening. In Figs. 8, there are regions of high $G$ along the curves marked by black dots, where the chemical potential in one graphene electrode coincides with the high density of states at the $n_{b, t}=0 \mathrm{LL}$ (and Dirac point energy) in that electrode. By contrast, when $B=0$, the density of states vanishes at the Dirac point and so $G=0$ when the chemical potential lies there [8]. 
We model our data (see Fig. 8b,d) using a Bardeen transfer-Hamiltonian approach, taking the full two component form of the LL eigenstates and the following device parameters: the doping densities in the bottom and top graphene layers are $2.0 \times 10^{11} \mathrm{~cm}^{-2}$ (p-type) and $3.6 \times 10^{11} \mathrm{~cm}^{-2}$ (n-type) respectively, and the twist angle $\theta=1^{\circ}$. We also include a Gaussian scattering potential with a spatial width of $\sigma=9 \mathrm{~nm}$, which arises from disorder in the graphene electrodes and allows small $(<\Delta K)$ $k$ changes that broaden the linewidth of the current peak (see Section 5 of the SI). A fit to the $I\left(V_{b}, V_{g}\right)$ curves at $B=0$ [8], provides accurate values of these parameters (see $\mathrm{SI})$.

Our model gives a good fit to the magneto-tunnelling data, in particular the shape and relative strength of the islands of high conductance. It enables a detailed analysis of the pattern of conductance peaks, which is dominated by the lateral overlap of the Landau states. The height of the boron nitride barrier affects the tunnel current magnitude but has little effect on the relative heights of the conductance peaks [5]. We now focus on the underlying physics that controls the overall pattern of peak amplitudes, in particular the effect of twist angle and chirality on the tunnelling process.

\section{Transition rates between chiral LL eigenstates}

The displacement, $\Delta K$, of the Dirac cones due to the twist angle is shown schematically in Figs. $4 \mathbf{a}, \mathbf{b}$. It can be represented by, and is equivalent to, the effect of a strong pseudo-magnetic field applied parallel to the graphene layers [14]. We describe the combined effects of the misalignment and the Landau-quantising applied magnetic field by a vector potential in the Landau gauge,

$$
\mathbf{A}_{b, t}=\left(l \hbar \Delta K_{x}^{ \pm},-e B x+l \hbar \Delta K_{y}^{ \pm}, 0\right) / e,
$$

where $l=0,1$ for the $\mathrm{b}$, $\mathrm{t}$ layers. In a perpendicular magnetic field, the electron wavefunctions at the $K^{+}$point have the analytic forms $[22,23]$

$$
\Psi_{n_{b, t}, k_{y, b, t}}^{K^{+}}(\mathbf{r}) \propto \exp \left(i k_{y, b, t}\right)\left(\begin{array}{c}
\phi_{\left|n_{b, t}\right|} \\
-\operatorname{sgn}\left(n_{b, t}\right) i \phi_{\left|n_{b, t}\right|-1}
\end{array}\right) .
$$

These two-component chiral states comprise plane waves along $y$, with wavenumber $k_{y, b, t}$ in the $\mathrm{b}$ and $\mathrm{t}$ layers, and simple harmonic oscillator ( $\mathrm{SHO})$ waves, $\phi$, along $x$ with indices that differ by 1 . The centres of the SHO wavefunctions in the top and bottom layers are shifted by $l_{B}^{2} \Delta K_{y}^{+}$and there is an additional plane wave factor for the top layer whose argument is $\Delta K_{x}^{+}\left(x-X_{t}\right)$, where $X_{t}=l_{B}^{2}\left(k_{y, t}+\Delta K_{y}^{+}\right)$. The Bloch states near the $K^{-}$ point have a similar form and make an equivalent contribution to the tunnelling matrix element, see SI. The tunnel rates between LLs, $W\left(n_{b}, n_{t}\right)$, depend on the overlap integrals of the initial and final wavefunctions summed

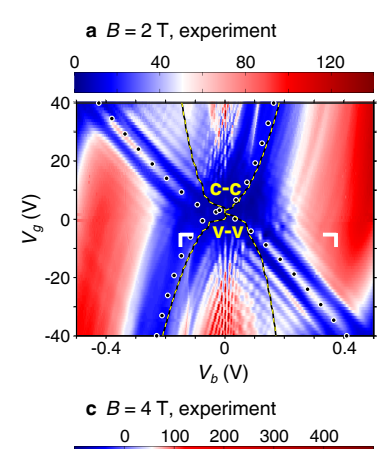

b $B=2 \mathrm{~T}$, theory

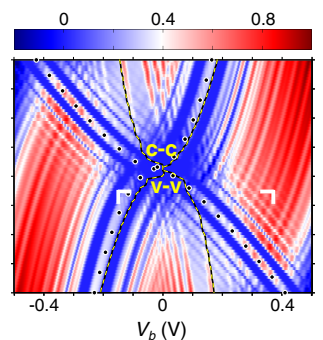

d $B=4 \mathrm{~T}$, theory
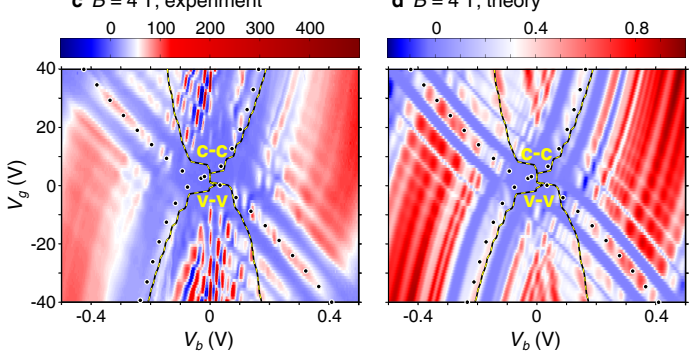

Figure 3: Differential magnetoconductance maps: experiment and theory. Colour maps showing $G\left(V_{b}, V_{g}\right)$ at $T=4 \mathrm{~K}$ measured (a) and calculated (b) when $B=2 \mathrm{~T}$ and (c measured, d calculated) when $B=4 \mathrm{~T}$. Colour scales for a,c are in $\mu \mathrm{S}$ and for $\mathbf{b}, \mathbf{d}$ normalised to the maximum conductance in the maps. Black and yellow dashed curves enclose regions around $V_{b}=0$ within which only conduction-conduction band (region labelled c-c with $V_{g}>0$ ), or only valence-valence band (region labelled v-v with $V_{g}<0$ ) tunnelling occurs. White right angles in $\mathbf{a}$ and $\mathbf{b}$ mark upper corners of the colour map regions enlarged in Fig. $5 \mathbf{a}$ and $\mathbf{b}$ respectively. Filled black circles running top left to bottom right (bottom left to top right) show loci along which the chemical potential the bottom (top) layer intersects with the Dirac point in that layer.

over the $k$-states in the two layers (see SI) and therefore permits tunnelling between SHO states with a range of different $n$ indices. Figs. $4 \mathbf{a}, \mathbf{b}$ show the energies and semiclassical trajectories (yellow rings) of the quantised Landau states.

Fig. 4c is a colour map of the inter-LL transition rate $W\left(n_{b}, n_{t}\right)$ at $B=4 \mathrm{~T}$ (see Eq. (25) of the SI). It reveals narrow yellow regions where $W\left(n_{b}, n_{t}\right)$ is high. In other areas (black), tunnelling is suppressed. The regions of high $W\left(n_{b}, n_{t}\right)$ originate from the spatial form and relative positions of the wavefunctions in the bottom and top electrodes. Within the upper right and lower left quadrants of the colour map, transitions between equivalent bands (conduction-conduction, c-c, and valence-valence, $\mathrm{v}-\mathrm{v})$ are strongly enhanced compared to tunnelling between different bands ( $\mathrm{c}-\mathrm{v}$ and $\mathrm{v}-\mathrm{c})$. This asymmetry, found for all values of $B$, is a consequence of the chiral nature of the electrons in graphene and arises from the interference of the electron wavefunction on the two sublattices of the bottom and top graphene layers. The figure shows that when the chirality of the electron is conserved i.e. for transitions between equivalent bands, tunnelling is enhanced. In contrast, when we remove the 
a

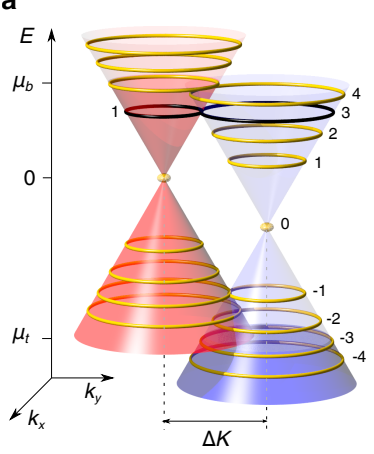

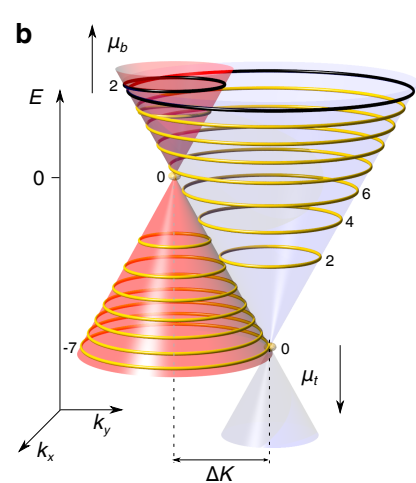

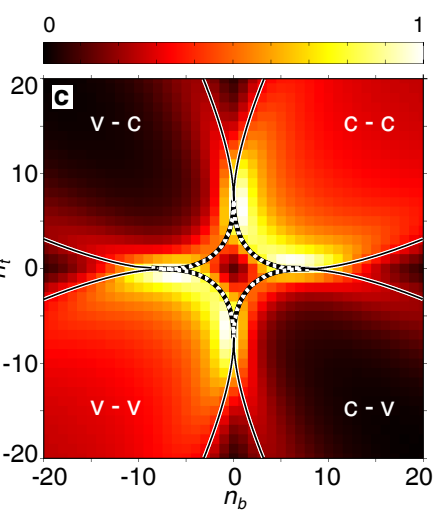

Figure 4: Energy alignment and tunnelling rates between the Landau levels of the two graphene electrodes. a,b Dirac cones showing the energy-wavevector dispersion relation, $E(\mathbf{k})$, for electrons in the bottom (red) and top (blue) graphene layers when $B=0$ and $V_{b}=0.28 \mathrm{~V}$ a and $0.58 \mathrm{~V} \mathrm{~b}$. Rings of constant energy on the surface of the cones show the energies and semiclassical $k$-space radii of LLs with indices $n_{b}$ and $n_{t}$. The black rings in $\mathbf{a}$ and $\mathbf{b}$ highlight $n_{b}=1$ to $n_{t}=3$ and $n_{b}=2$ to $n_{t}=16$ transitions, respectively. Occupied electron states in the bottom (top) layer are shaded dark red (blue) up to the Fermi level, $\mu_{b, t}$, in that layer. c Colour map showing tunnelling rates, $W\left(n_{b}, n_{t}\right)$, normalized to the maximum rate in the plot, for scattering-assisted transitions (details in SI) between LLs with indices $n_{b}$ and $n_{t}$ in the bottom and top electrodes. The dotted and solid curves show the loci calculated using Eq. (3). For all panels, $B=4 \mathrm{~T}$.

effect of chirality from our model by using pure (single component) LL wavefunctions, the tunnelling matrix elements are the same for transitions between equivalent and different bands (see SI).

\section{Effect of chirality on tunnel current}

The asymmetry in the transition rate colour map in Fig. 4c manifests itself in the observed pattern of conductance peak amplitudes. In certain regions of the $\left(V_{b}, V_{g}\right)$ plot, tunnelling is exclusively between equivalent bands, as shown in Fig. 8. Here, the black and yellow dashed curves bound the regions of $V_{b}-V_{g}$ space where tunnelling is either only c-c (upper region, $V_{g}>0$ ) or v-v (lower region, $V_{g}<0$ ), respectively. Within these regions the amplitudes of most resonant peaks are high, i.e. dark red. Increasing $V_{b}$ beyond the lower region induces a changeover from tunnelling between equivalent bands to a mixture of tunnelling between equivalent and different bands and is therefore accompanied by a suppression of the conductance peaks. This is a direct manifestation of electron chirality. A similar changeover occurs as $V_{b}$ decreases across the left hand edge of the region bounded by the upper dashed curve in Fig. 8a-d.

The effect of chirality on the peak amplitudes in these regions is seen more clearly in the enlarged lower region of the $G\left(V_{b}, V_{g}\right)$ maps at $B=2 \mathrm{~T}$ shown in Figs. 5a-c. In both our experiment, $\mathbf{a}$, and calculations, $\mathbf{b}$, the conductance peak amplitudes are larger within the bounded region in the lower left-hand side of the plot, labelled $\mathrm{L}$ in Fig. 5d, where v-v tunnelling dominates and smaller in the bounded region in the lower right-hand side of the plot where tunnelling is a mixture of $\mathrm{v}-\mathrm{v}$ and $\mathrm{v}-\mathrm{c}$ transitions (region labelled R in Fig. 5d). For comparison, in Fig. $5 \mathbf{c}$ we show $G\left(V_{b}, V_{g}\right)$ calculated when chirality is "switched off", i.e. with each eigenstate represented by a single SHO wavefunction with no pseudospin component (see SI). In contrast to the chiral theory and experimental data, the conductance peaks for the non-chiral calculations have similar amplitudes in regions $\mathrm{L}(\mathrm{v}-\mathrm{v})$ and $\mathrm{R}$ ( $\mathrm{v}-\mathrm{v}$ and $\mathrm{v}-\mathrm{c})$.

To quantify the effect of chirality on the tunnel current, we calculate the ratio of the mean conductance in region $\mathrm{L}$ to that in region $\mathrm{R},\langle G\rangle_{L} /\langle G\rangle_{R}$ (see Fig. 5d). In the bar chart in Fig. 5e we show $\langle G\rangle_{L} /\langle G\rangle_{R}$ when $B=0$, 2 and 4 T. For each field value, $\langle G\rangle_{L} /\langle G\rangle_{R}$ for the measured data (red) and the chiral calculations (yellow) are similar to each other. In contrast $\langle G\rangle_{L} /\langle G\rangle_{R}$ is significantly smaller for the non-chiral calculations (blue). In addition, with increasing $B$ the difference between the chiral and non-chiral results becomes larger: at higher $B$ there are fewer LL transitions within regions $\mathrm{L}$ and $\mathrm{R}$ and, for those transitions that do occur, the difference between the chiral and non-chiral conductance is more pronounced. Hence, the measured dependence of the conductance peak amplitudes on $V_{g}, V_{b}$, and $B$ reveals and demonstrates the chiral nature of the electrons and the associated asymmetry in the tunnelling rates (see Fig. 4c).

\section{Nested and figure of 8 cyclotron orbits}

A semiclassical picture, in which electrons undergo cyclotron motion in both real- and $k$-space, provides further insights into the physics of tunnelling in these devices. In $k$-space, the orbital radii in the two graphene layers are $\kappa_{b, t}=\sqrt{2\left|n_{b, t}\right|} / l_{B}$ and the orbit centres are separated by $\boldsymbol{\Delta} \mathbf{K}^{ \pm}$. The solid and dotted curves in Fig. 4c are loci 
of initial and final states along which the corresponding semiclassical orbits just touch, so that the tunnelling electrons can make a continuous classical trajectory in the $\left(k_{x}, k_{y}\right)$ plane. These loci are defined by

$$
\kappa_{t}=\Delta K(\theta) \pm \kappa_{b}
$$

Here, the - and + signs specify, respectively, cyclotron orbits that describe a "figure of 8" (F-8) and nested (N) form. Examples are shown by the projected circles in the lower parts of Figs. $6 \mathbf{a}$ and $\mathbf{b}$. The spatial variation of the real (dark) and imaginary (light) components of the corresponding two-component LL wavefunctions are also shown ( $x$ axis re-scaled by $1 / l_{B}^{2}$ to enable comparison between the k-space trajectories and the spatial form of the SHO wavefunctions). The maxima in the wavefunction amplitude are located at the turning points of the semiclassical orbit so that, when Eq. (3) is satisfied, i.e. along the solid (dotted) locus in Fig. 4c for N (F-8) orbits, the wavefunction overlap integral is large. This large spatial overlap produces the bright yellow regions of high tunnelling rates in Fig. 4c.

The twist angle of the graphene electrodes determines the value of $\Delta K(\theta)$ in Eq. (3) and thus shapes the boundary loci of the four distinct regions (c-c, v-v, v-c, c-v). The twist angle also manifests itself through the suppression of the 0-0 transition at the centre of the plot, because the spatial displacement of the two states exceeds their spatial width. The scattering potential broadens the variation of the tunnelling rates with $n_{b}$ and $n_{t}$ but preserves the effects of chirality and of the spatial wavefunction overlap on the overall form of the plot (see SI).

The $\mathrm{N}$ and F-8 semiclassical orbits determine the dependence of $G$ on $B, V_{b}$ and $V_{g}$. At the onset of current (see red curve and arrow labelled $V_{1}$ in Fig. 2a) the energetically aligned LLs correspond to semiclassical orbits with the F-8 form, see black rings in Fig. 4a. Consequently the matrix elements are large, allowing tunnel current to flow. At the resonant current peak (see red curve and arrow labelled $V_{2}$ in Fig. 2a) the Dirac cones just touch and their intersection is a straight line. As a result, all energetically aligned LLs have high matrix elements because all the corresponding semiclassical orbits have either F-8 or $\mathrm{N}$ forms, see black and yellow rings in Fig. 4b. When $V_{b}$ increases beyond the current peak, many LLs that become aligned energetically have cyclotron orbits that do not overlap spatially and so the tunnelling matrix elements and current decreases.

The semiclassical analysis also highlights the effect of the lattice misalignment on the electron dynamics. At the point of intersection of the $n_{b}=1$ and $n_{t}=3$ orbits, the electron "back-scatters" in both $k$-space and real space, making a $180^{\circ}$ direction change where the orbits touch in the $\left(k_{x}-k_{y}\right)$ plane, see lower part of Fig. $6 \mathbf{a}$. This change in kinetic momentum at the intersection between the two orbits is induced by the impulse, $\hbar \Delta \mathbf{K}^{ \pm}$, arising from the misorientation of the two graphene layers
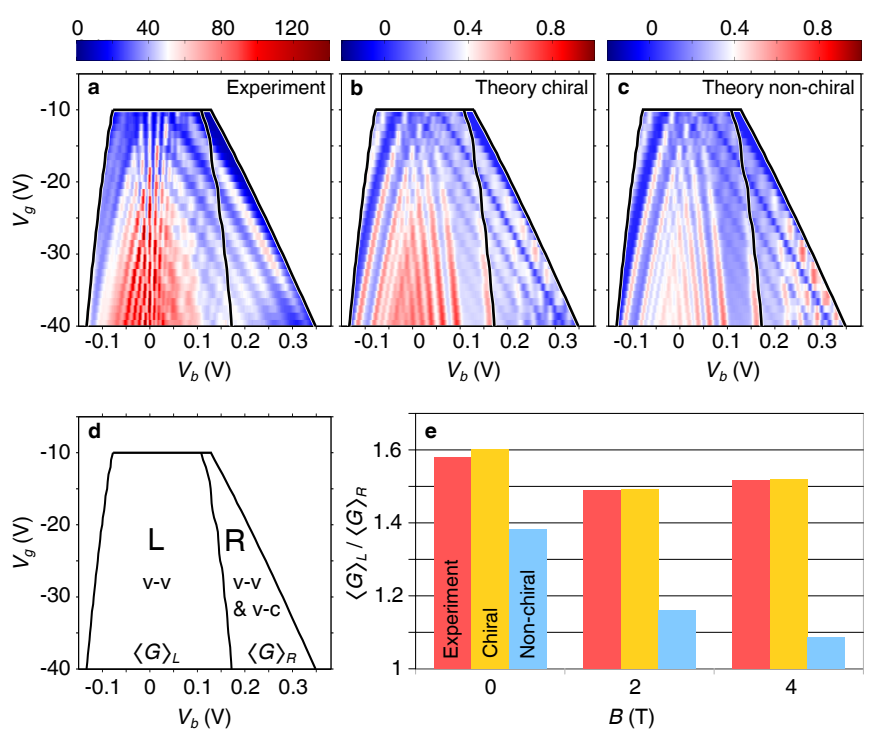

Figure 5: Effect of chirality on the differential magnetoconductance: experiment and theory. a-c Colour maps showing $G\left(V_{b}, V_{g}\right)$ for $B=2 \mathrm{~T}$. a, $\mathbf{b}$ are enlargements of the lower parts of the colour maps in Fig. 8a, $\mathbf{b}$ respectively (defined by white right angles). Panel a shows experimental data $(T=4 \mathrm{~K}), \mathbf{b}$ is calculated using the full model with chiral electrons, and $\mathbf{c}$ calculated using non-chiral wavefunctions i.e. each comprising a single simple harmonic oscillator state. Colour bars in a and $\mathbf{b}, \mathbf{c}$ are in $\mu \mathrm{S}$ and normalised units, respectively. Solid curves in a-c enclose regions of the colour map where tunnelling is only $\mathrm{v}-\mathrm{v}$ (labelled $\mathrm{L}$ in $\mathbf{d}$ ) or a mixture of $\mathrm{v}-\mathrm{v}$ and $\mathrm{v}-\mathrm{c}$ (labelled $\mathrm{R}$ in $\mathbf{d})$. Bar charts in e show the ratio, $\langle G\rangle_{L} /\langle G\rangle_{R}$, of the mean conductance in regions $\mathrm{L}$ and $\mathrm{R}$ (see $\mathbf{d}$ ) for the measured data (red), and calculated for chiral (yellow) and non-chiral (blue) electrons.

and the associated vector potential, which acts like an inplane pseudo-magnetic field for tunnelling electrons, see Eq. (4).

As shown in Fig. 6a, for the F-8 orbits, the tunnelling transition reverses the wavevector in the bottom and top electrodes, $\mathbf{k}_{b}$ and $\mathbf{k}_{t}$, measured relative to the Dirac point of the two layers (where $\left|\mathbf{k}_{b, t}\right|=\kappa_{b, t}$ ). In contrast, for $\mathrm{N}$ orbits the direction of the wavevector in the two electrodes is unchanged during tunnelling; only its magnitude changes (Fig. 6b).

\section{Cyclotron orbits and Klein tunnelling}

In graphene, the chiral nature of an electron in the absence of a magnetic field can be expressed by the expectation value of the pseudospin operator with respect to the eigenstate. For the $K^{ \pm}$valley this expectation value is $\langle\boldsymbol{\sigma}\rangle=s( \pm \cos \varphi, \sin \varphi)$, where $\varphi$ is the polar direction of the wavevector. When $B \neq 0,\langle\boldsymbol{\sigma}\rangle$ can be defined semiclassically in a similar way (see Section 5 of the SI). Consequently, in our semiclassical model, for $\mathrm{N}$ orbits in both valleys $\langle\boldsymbol{\sigma}\rangle$ is unchanged for equivalent band transitions but is rotated by $180^{\circ}$ for transitions 


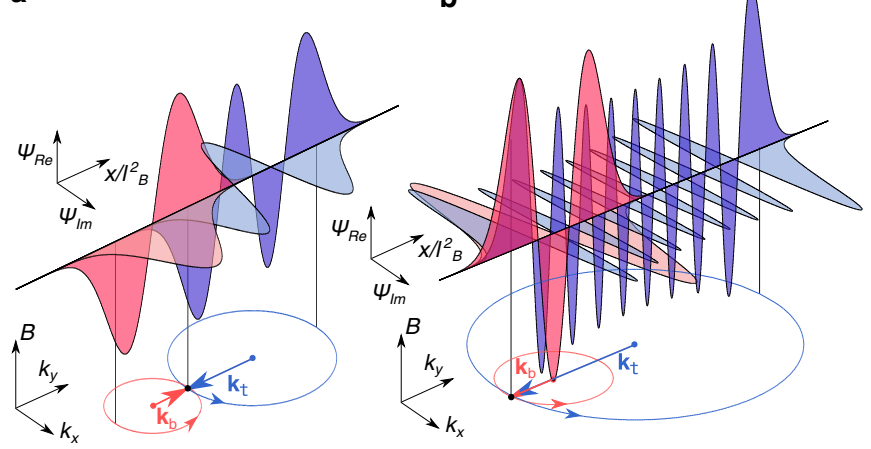

Figure 6: Electron wavefunctions and semiclassical cyclotron orbits in the two graphene layers for figure-of- 8 and nested tunnelling transitions. a,b Upper: vertical (horizontal) curves show the real (imaginary) parts of the real space electron wavefunction in the bottom (red curves) and top (blue curves) graphene electrodes respectively with $B=4 \mathrm{~T}$ and $\mathbf{a} n_{b}=1$ (red) and $n_{t}=3$ (blue) and $\mathbf{b} n_{b}=2$ (red) and $n_{t}=16$ (blue). The $x$ axis is scaled by $l_{B}^{2}$ for comparison with lower plots: circles show corresponding figure of 8 and nested cyclotron orbits in $k$ - space $\left(k_{x}, k_{y}\right.$ axes inset and direction of motion marked by arrows) with orbit centres separated by $\Delta K$. The vertical black lines connecting upper and lower parts of the figure show the classical turning points.

between different bands. In contrast, for F-8 orbits $\langle\boldsymbol{\sigma}\rangle$ is reversed for transitions between equivalent bands and unchanged for transitions between different bands.

When $\langle\boldsymbol{\sigma}\rangle$ is unchanged, the inter-layer tunnelling process bears an analogy with intra-layer Klein tunnelling [1-3]. The Klein paradox is predicted to occur for electrons tunnelling through a barrier in planar graphene where unity transmission is expected when the pseudospin is conserved. In our device, the tunnelling electron makes a "quantum jump" across the barrier; hence, the tunnelling rate can be high even if pseudospin is reversed, provided there is strong spatial overlap between the initial and final LL wavefunctions. However, as for the case of Klein tunnelling in planar graphene, the orientation of $\langle\boldsymbol{\sigma}\rangle$ in the initial and final states determines the tunnelling rate (see Section 6 of the SI). Physically this is due to the interference between the two sublattices of the two graphene layers (see Eq. (13) of the SI and [1]). In our experiments, resonant tunnelling is enabled by the twist of the graphene electrodes. This provides the impulse to induce the momentum and orbit centre change required for energy- and $\mathbf{k}$-conserving tunnel transitions with high matrix elements. In particular, our data indicate that the pseudospin of the electrons is conserved for the tunnelling transitions at the current peak.

\section{Conclusions}

Our results show that the chirality can play an important role in determining the electronic characteristics of graphene-based vertical devices. This property repre- sents a degree of freedom additional to those which arise from electron spin and valley degeneracies and, therefore, should be taken into account when assessing possible performance of electronic components based on materials with Dirac-like energy spectra.

\section{Acknowledgments}

This work was supported by the EU Graphene Flagship Programme and ERC Synergy Grant, Hetero2D. M.T.G. acknowledges The Leverhulme Trust for support of an Early Career Fellowship. V.I.F. acknowledges support of a Royal Society Wolfson Research Merit Award. E.E.V. and S.V.M. were supported by NUST "MISiS" (grant K1-2015-046) and RFBR (15-02-01221 and 14-0200792).

\section{Author contributions}

Y.C., A.V.K., fabricated the devices. E.E.V., A.M., O.M., A.P., K.S.N., A.V.K., M.J.Z., L.E., designed and/or carried out the experiments. M.T.G., T.M.F., L.E., E.E.V., A.M., S.V.M., J.R.W., V.I.F., K.S.N., A.K.G., undertook the interpretation of the data. M.T.G. performed the calculations. M.T.G., T.M.F., and L.E. wrote the manuscript with contributions from the other authors.

\section{Methods}

The device fabrication is described in the methods section of ref. [8]. 


\section{SUPPLEMENTARY INFORMATION}

\section{DEVICE GEOMETRY}

A schematic diagram of the device is presented in Fig. 1 of the main text and an optical micrograph is shown in Fig. 1 of this document. Our device consists of two single layer graphene electrodes separated by a $1.4 \mathrm{~nm}$ thick boron nitride $(\mathrm{hBN})$ tunnel barrier. The bottom graphene layer is mounted on an atomically flat hBN layer which, in turn, rests on the oxidised surface of a $\mathrm{SiO}_{2} / \mathrm{n}-\mathrm{Si}$ substrate. A capping layer of hBN covers the upper graphene layer. These three hBN layers are not intentionally aligned with the graphene layers. The device is made using the standard dry transfer proceedure outlined in [5] but with the additional step that the lattices of the two graphene layers are aligned to within $1^{\circ}$, as described in [8], which reports studies of the same device in zero magnetic field.

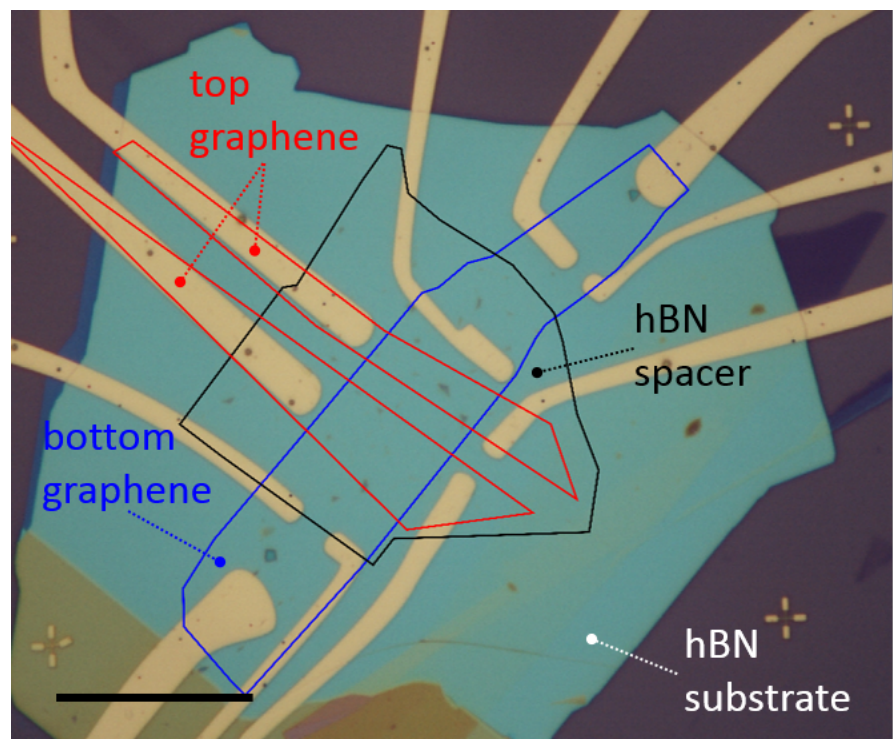

Figure 7: Optical micrograph of our structure, which consists of two tunnelling devices whose "active" regions correspond to the overlap of the regions indicated by the red and blue lines. The results reported in the paper are for the device with overlapping layers on the lower left side, which has an active area of $\approx 100 \mu \mathrm{m}^{2}$. The top and bottom graphene layers are crystallographically aligned to each other to $\approx 1^{\circ}$. Bottom left: $25 \mu \mathrm{m}$ scale bar.

High quality devices are required in order to reveal the subtle features in the inter-Landau level tunnelling data. We have measured four different resonant tunnelling diodes (RTDs) of this type. In addition to the one described in the present manuscript, two others were of similarly high quality. In this paper we present data from a device in which the graphene lattices were the most closely aligned and consequently reveal the effects of chirality most clearly.

\section{MODEL}

The graphene lattices in our device are slightly misorientated by an angle $\theta \approx 1^{\circ}$ which results in a relative displacement in the positions of the Dirac points in $K$ space, $\Delta \mathbf{K}^{ \pm}=(R(\theta)-1) \mathbf{K}^{ \pm}$, where $R(\theta)$ is the rotation matrix. The label \pm corresponds to the two inequivalent $K$ points with positions given by $\mathbf{K}^{ \pm}= \pm(4 \pi / 3 a, 0)$, where $a=2.46 \AA$ is the lattice constant of graphene. The Dirac points in the bottom electrode are at $\mathbf{K}_{b}^{ \pm}$and in the top electrode $\mathbf{K}_{t}^{ \pm}+\Delta \mathbf{K}^{ \pm}$. The relative shift of the Dirac points is analogous to an in-plane magnetic field. Therefore, we describe the displacement of the $K$ points using the following vector potential for electrons in the bottom and top layers, which also includes the effect of a magnetic field, $\mathbf{B}$ that is applied perpendicular to the graphene layers,

$$
\mathbf{A}_{b, t}=\left(l \hbar \Delta K_{x}^{ \pm},-e B x+l \hbar \Delta K_{y}^{ \pm}, 0\right) / e,
$$

where $l=0,1$ in the bottom (b) and top (t) layers respectively. We can therefore write the magnitude of the effective magnetic field due to the misorientation of the layers as $B_{\text {eff }}=\hbar|\boldsymbol{\Delta K}| / e d=140 \mathrm{~T}$ where $d=1.4 \mathrm{~nm}$ is the $\mathrm{hBN}$ barrier width. The electron momentum takes the form $\mathbf{p} \rightarrow \mathbf{p}+e \mathbf{A}$, so that the effective mass Hamiltonian for Dirac electrons in graphene becomes 


$$
H_{b, t}^{ \pm}=v_{F}\left(\begin{array}{cc}
0 & \pm\left(p_{x}+e A_{x, b, t}\right)-i\left(p_{y}+e A_{y, b, t}\right) \\
\pm\left(p_{x}+e A_{x, b, t}\right)+i\left(p_{y}+e A_{y, b, t}\right) & 0
\end{array}\right)
$$

where $v_{F}=10^{6} \mathrm{~ms}^{-1}$. The Hamiltonian has the form of a quantum harmonic oscillator so that the electron has discrete Landau energy levels given by

$$
E_{n_{b, t}}^{2}=\operatorname{sgn}\left(n_{b, t}\right)\left|n_{b, t}\right| 2 e B \hbar v_{F}^{2},
$$

where $n_{b, t}$ is an integer that labels the energy levels in the two electrodes, positive for electrons in the conduction band and negative in the valence band and

$$
\operatorname{sgn}(n)= \begin{cases}1 & (n>0) \\ 0 & (n=0) \\ -1 & (n<0)\end{cases}
$$

The electron wavefunctions at the two Dirac points are therefore

$$
\Psi_{n_{b, t}, k_{y, b, t}}^{K^{+}}(\mathbf{r})=\frac{C_{n_{b, t}}}{\sqrt{L}} \exp \left(i k_{y, b, t} y\right)\left(\begin{array}{c}
\phi_{\left|n_{b, t}\right|} \\
-\operatorname{sgn}\left(n_{b, t}\right) i \phi_{\left|n_{b, t}\right|-1}
\end{array}\right)
$$

and

$$
\Psi_{n_{b, t}, k_{y, b, t}}^{K^{-}}(\mathbf{r})=\frac{C_{n_{b, t}}}{\sqrt{L}} \exp \left(i k_{y, b, t} y\right)\left(\begin{array}{c}
\operatorname{sgn}\left(n_{b, t}\right) i \phi_{\left|n_{b, t}\right|-1} \\
\phi_{\left|n_{b, t}\right|}
\end{array}\right)
$$

where

$$
C_{n}= \begin{cases}1 & (n=0) \\ 1 / \sqrt{2} & (n \neq 0)\end{cases}
$$

where

$$
\phi_{\left|n_{b}\right|}=\frac{1}{\sqrt{2^{\left|n_{b}\right|}\left|n_{b}\right| ! \sqrt{\pi} l_{B}}} \exp \left[-\frac{1}{2 l_{B}^{2}}\left(x-X_{b}\right)^{2}\right] H_{\left|n_{b}\right|}\left(\frac{1}{l_{B}}\left(x-X_{b}\right)\right)
$$

and

$$
\phi_{\left|n_{t}\right|}=\frac{1}{\sqrt{2^{\left|n_{t}\right|}\left|n_{t}\right| ! \sqrt{\pi} l_{B}}} \exp \left[-\frac{1}{2 l_{B}^{2}}\left(x-X_{t}\right)^{2}-i \Delta K_{x}^{ \pm}\left(x-X_{t}\right)\right] H_{\left|n_{t}\right|}\left(\frac{1}{l_{B}}\left(x-X_{t}\right)\right),
$$

Here $l_{B}=\sqrt{\hbar / e B}$ and $H_{n}$ is the nth order Hermite polynomial. These two-component chiral states comprise plane waves along $y$, with wavenumber $k_{y, b, t}$ in the $\mathrm{b}$ and t layers, and simple harmonic oscillator (SHO) waves, $\phi$, along $x$ with indices that differ by 1 . The orbit centre in the bottom and top electrodes are given by $X_{b}=l_{B}^{2} k_{y, b}$ and $X_{t}=l_{B}^{2}\left(k_{y, t}+\Delta K_{y}^{ \pm}\right)$respectively. The effect of the misorientation of the two graphene sheets is to shift the relative position of their orbit centres by $l_{B}^{2} \Delta K_{y}^{ \pm}$and introduce a phase difference of $\Delta K_{x}\left(x-X_{t}\right)$.

\section{Matrix element}

We assume that electrons can undergo elastic scattering which we describe using a Gaussian scattering potential:

$$
V_{S}(x, y)=V_{0} e^{-x^{2} / 2 \sigma_{s}^{2}-y^{2} / 2 \sigma_{s}^{2}}
$$


where $\sigma_{s} \approx 10 \mathrm{~nm}$ is the scattering length scale. This value of $\sigma_{s}$ agrees with values reported in the literature (Ref. [6] and references therein) and gives the best fit to the line width of the peak in the current-voltage curve when $B=0$ [8]. The matrix element for tunnelling between the bottom and top electrodes is given by

$$
M_{b t}=\int_{V} d V \Psi_{t}^{*}(\mathbf{r}, z) V_{S} \Psi_{b}(\mathbf{r}, z) .
$$

First we consider the integral in the $z$ direction. We assume that the electron wavefunctions decay exponentially into the barrier regions so that the integral is a constant, equal to

$$
\Xi=\frac{V_{0}}{D} e^{-\kappa d}
$$

where $d$ is the barrier width. We assume $\kappa$ to be independent of energy to facilitate analysis of the current. For full analysis of different $V_{b}$ dependent models for $\kappa$, see Ref. [5]. In the basis of Bloch wavefunctions [8, 9], the matrix element is given by

$$
\begin{aligned}
M_{b t}\left(n_{b}, n_{t}, k_{y, b}, k_{y, t}\right)= & \frac{1}{L} C_{n_{b}} C_{n_{t}} \Xi I_{y}\left(k_{y, b}, k_{y, t}\right)\left[I_{x}\left(\left|n_{b}\right|,\left|n_{t}\right|, k_{y, b}, k_{y, t}\right) \mp i \operatorname{sgn}\left(n_{b}\right) I_{x}\left(\left|n_{b}\right|-1,\left|n_{t}\right|, k_{y, b}, k_{y, t}\right)\right. \\
& \left. \pm i \operatorname{sgn}\left(n_{b}\right) I_{x}\left(\left|n_{b}\right|,\left|n_{t}\right|-1, k_{y, b}, k_{y, t}\right)+\operatorname{sgn}\left(n_{b}\right) \operatorname{sgn}\left(n_{T}\right) I_{x}\left(\left|n_{b}\right|-1,\left|n_{t}\right|-1, k_{y, b}, k_{y, t}\right)\right]
\end{aligned}
$$

where $I_{x}$ and $I_{y}$ are the overlap integrals of the wavefunctions along the $x$ and $y$ axes respectively. On first inspection, Eq. (16) appears to reveal that the matrix element is different for tunnelling between $K^{+}$valleys (upper sign) compared to that between $K^{-}$valleys (lower sign). However, $\Delta \mathbf{K}^{+}=-\Delta \mathbf{K}^{-}$and, consequently, it can be shown that the matrix element for transitions between the same valleys are equivalent. Our matrix element does not explicitly include the cell-periodic parts of the Bloch functions, $u^{\alpha, \beta}(\mathbf{r})$, where $\alpha$ and $\beta$ label the two atoms in grahene's unit cell. This is because for small relative rotations of the two layers, the spatial overlap integral of the cell-periodic parts of the wavefunction $\int d S u^{* \alpha, \beta}(R(\theta) \mathbf{r}) u^{\alpha, \beta}(\mathbf{r})$ are approximately equivalent for all combinations of $\alpha$ and $\beta$, and therefore will only have a small quantitative effect on the matrix element [9].

\section{Overlap integrals for scattering assisted tunnelling}

The overlap integrals $I_{y}$ and $I_{x}$ can be shown [33] to have following form:

$$
I_{y}=\sqrt{2 \pi} \sigma_{s} \exp \left(-\Delta k^{2} \sigma_{s}^{2} / 2\right)
$$

within which $\Delta k=k_{y, b}-k_{y, t}$. The overlap integral in the $x$ direction, $I_{x}$, is given by:

$$
\begin{gathered}
I_{x}\left(n_{b}, n_{t}, k_{y, b}, k_{y, t}\right)=\frac{1}{\zeta l_{B}} P_{b t}\left(n_{b}, n_{t}, k_{y, b}, k_{y, t}\right) \sum_{j=0}^{\left\lfloor n_{b}, n_{t}\right\rfloor} j !\left(\begin{array}{c}
n_{b} \\
j
\end{array}\right)\left(\begin{array}{c}
n_{t} \\
j
\end{array}\right)\left(1-a^{2}\right)^{\left(n_{b}+n_{t}\right) / 2-j} \times \\
\left(2 a^{2}\right)^{j} H_{n_{b}-j}\left[\frac{a \Upsilon-l_{B} k_{y, b}}{\left(1-a^{2}\right)^{1 / 2}}\right] H_{n_{t}-j}\left[\frac{a \Upsilon-l_{B}\left(k_{y, t}+\Delta K_{y}^{+}\right)}{\left(1-a^{2}\right)^{1 / 2}}\right]
\end{gathered}
$$

where $a=1 / \zeta l_{B}$,

$$
\begin{gathered}
\zeta^{2}=\left(\frac{1}{l_{B}^{2}}+\frac{1}{2 \sigma_{s}^{2}}\right), \\
P_{b t}\left(n_{b}, n_{t}, k_{y, b, t}\right)=\frac{\exp \left[\vartheta\left(k_{y, b}, k_{y, t}\right)\right]}{\sqrt{2^{n_{t}} n_{t} ! 2^{n_{b}} n_{b} !}},
\end{gathered}
$$


within which

$$
\vartheta=\Upsilon^{2}-\frac{l_{B}^{2}}{2}\left(\left(k_{y, t}+\Delta K_{y}^{ \pm}\right)^{2}+k_{y, b}^{2}\right)-i \Delta K_{x}^{ \pm}\left(k_{y, t}+\Delta K_{y}^{ \pm}\right)
$$

and

$$
\Upsilon=\frac{1}{2 \zeta}\left(k_{y, t}+\Delta K_{y}^{ \pm}+k_{y, b}+i \Delta K_{x}^{ \pm}\right)
$$

\section{Current}

\section{Expression for the tunnel current}

The current between the layers is given by the sum over states in the top and bottom layers:

$$
I=g_{V} \frac{4 \pi e}{\hbar} \sum_{b t}\left|M_{b t}\right|^{2}\left[f_{b}\left(E_{b}\right)-f_{t}\left(E_{t}\right)\right] \delta\left(E_{b}-E_{t}\right),
$$

where the Fermi functions for the bottom and top layers are given, respectively, by

$$
f_{b}\left(E_{b}\right)=\frac{1}{1+e^{\left(E_{b}-\mu_{b}\right) / k_{B} T}}
$$

and

$$
f_{t}\left(E_{t}\right)=\frac{1}{1+e^{\left(E_{t}-\mu_{t}\right) / k_{B} T}} .
$$

and $k_{B} T$ is the thermal energy. We assume that the Landau levels (LLs) are broadened in energy by $\Gamma_{b, t}$ in the bottom and top electrodes respectively due to electron - electron interactions, which we model with a set of Gaussian functions (to ensure convergence at low magnetic fields) centered on the energies of the LLs $E_{n}$ (see Eq. (6)) [21]

$$
\Gamma(E)=\sum_{n=-\infty}^{\infty} \frac{1}{\sqrt{2 \pi} \Gamma_{b, t}} \exp \left(-\frac{\left(E-E_{n}\right)^{2}}{2 \Gamma_{b, t}^{2}}\right) .
$$

The density of states is then given by $D(E)=\left(2 / \pi l_{B}^{2}\right) \Gamma(E)$. We convert the sum over $k$-states in Eq. (24) to an integral to find the contribution to the current for transitions between LLs $n_{t}$ and $n_{b}$ is given by

$$
W\left(n_{b}, n_{t}\right)=\frac{2 L^{4}}{\pi^{2} l_{B}^{4}} \iint\left|M_{b t}\right|^{2} d k_{y, b} d k_{y, t}
$$

where $L$ is the device length, so that after using the $\delta$ function to integrate out $E_{t}$, we find that the current can now be expressed by:

$$
I=g_{V} \frac{4 \pi e}{\hbar} \int W\left(n_{b}, n_{t}\right)\left[f_{b}\left(E_{b}\right)-f_{t}\left(E_{t}\right)\right] D_{b}\left(E_{b}\right) D_{t}\left(E_{b}-\phi\right) d E_{b}
$$

Form of the tunnel current versus bias voltage curve for $B=0$

A detailed analysis of the form of the $I\left(V_{b}\right)$ curve for $B=0$ is given in Ref. [8]. In the Supplementary Video (SV) we present an animation, which shows how the key features in the measured (red) and modeled, with momentum conserving transitions only $\left(\sigma_{s} \rightarrow \infty\right.$, blue), $I\left(V_{b}\right)$ curves relate to the form of the intersection (shown yellow in the $\mathrm{SV}$ ) between the two Dirac cones as they move relative to one another in response to changes in $V_{b}$. 


\section{Electrostatic model}

We model the electrostatics, i.e. the values of $\mu_{b, t}$ and the electrostatic potential energy difference $\phi_{b}$ between the graphene layers, by solving the following equation:

$$
\phi+\mu_{t}\left(\rho_{t}, \Gamma_{t}\right)-\mu_{b}\left(\rho_{b}, \Gamma_{b}\right)+e V_{b}=0
$$

where $d=1.4 \mathrm{~nm}$ is the barrier width, $\rho_{b, t}$ is the charge density on the bottom and top electrodes and the function $\mu(\rho, \Gamma)$ is found using the density of states, $D(E)[5]$. From Gauss's law, and ensuring charge neutrality, we obtain the following relationships between $V_{b}, V_{g}, \phi$ and $n_{b, t}$ :

$$
\begin{array}{r}
\epsilon\left(F_{b}-F_{g}\right)=\rho_{b} \\
-\epsilon F_{b}=\rho_{t},
\end{array}
$$

where $F_{b}=\phi_{b} / e d$ and $F_{g}=\left(e V_{g}-\mu_{b}\right) / e D_{g}$ are the fields in the tunnel barrier and gate-oxide barrier respectively and $D_{g}=300 \mathrm{~nm}$ is the oxide thickness.

\section{ANALYSIS OF CONDUCTANCE PEAKS}

Fig. 8 shows colour maps of $G\left(V_{b}, V_{g}\right)=d I / d V_{b}$ measured $(\mathbf{a}, \mathbf{c})$ and calculated $(\mathbf{b}, \mathbf{d})$ when $B=2 \mathrm{~T}(\mathbf{a}, \mathbf{b})$ and $B=4 \mathrm{~T}(\mathbf{c}, \mathbf{d})$. The parameters used to model the measured data are $\sigma_{s}=9 \mathrm{~nm}$ and the LL broadening in the bottom and top graphene electrodes, $\Gamma_{b}$ and $\Gamma_{t}$, is set at $4 \mathrm{meV}$ and $4 \mathrm{meV}(6 \mathrm{meV}$ and $8 \mathrm{meV})$ respectively when $B=2 \mathrm{~T}(4$ $\mathrm{T})$. Note that we focus on magnetic field values $\leq 4 \mathrm{~T}$ because higher fields lead to a strong in-plane magnetoresistance in the sections of the graphene layers that carry the current to the active tunnelling area of the device. Although resonant peaks due to inter-Landau level tunnelling are observed at these higher fields, their voltage positions are significantly displaced due to the magnetoresistance, making it difficult to interpret the data and compare it with theory.

In this section we explain in more detail the origin of the conductance peaks observed in $G\left(V_{b}, V_{g}\right)$. The filled black circles in Fig. 8 show the calculated $\left(V_{b}, V_{g}\right)$ loci for which the chemical potential in the top layer intersects with the zeroth LL in that layer, see inset $\mathbf{A}$ (filled circles running bottom left to top right), and for which the chemical potential in the bottom layer coincides with the zeroth LL in the bottom layer, see inset $\mathbf{B}$ (filled circles running top left to bottom right). Therefore, the local conductance peaks that lie along the X-shaped loci correspond to the alignment of the chemical potential in one graphene layer with the peak in the density of states for the LL at the Dirac point.

Fig. 8 shows that in both our experiments and theory, when $V_{g} \approx 5 \mathrm{~V}$ and $V_{b} \lesssim 0.2 \mathrm{~V}$, increasing $V_{b}$ initially has little effect on $G$. But when $V_{b} \approx \pm 0.2 \mathrm{~V}$, there is a sharp increase in conductance. When $V_{b}$ increases beyond $\approx 0.5$ $\mathrm{V}, G$ decreases, becoming negative after the peak in $I\left(V_{b}\right)$. The regions of high $G$ in Fig. 8 form stripe patterns with similar shapes to the loci marked by the filled circles. This is because they also originate from alignment of the chemical potential and LLs when $\mu_{b, t}=E_{n_{b, t}}$ where, in contrast to the yellow curves, $n_{b, t} \neq 0$. The crossing of these loci gives rise to more islands of high $G$, for example those labelled "B-D" in Fig. 8 c,d.

When $B=4 \mathrm{~T}$, we find good qualitative agreement between the measured and calculated $G\left(V_{b}, V_{g}\right)$ colour maps. Along the loci marked by filled circles in Fig. 8c both maps reveal a series of conductance maxima in similar positions, for example those labelled "B-D" in Fig. 8c and $\mathbf{d}$. As explained above, along the loci, the maxima occur as $\mu_{t}$ sweeps through the LL spectra in the top and bottom layers. The maxima labelled "B" and "C" occur when $\mu_{t}$ coincides with $n_{t}=-1$ and $n_{t}=-2$ LLs (see insets labelled "B" and "C"). The strength of the maxima depends on the alignment of the LLs. For example, the conductance maximum labelled "D" is stronger than "B", because at "B" the LL spectra in the top and bottom layers are aligned and tunnelling occurs from $n_{b}=0$ and -1 to $n_{t}=0$ and -1 , which have low matrix elements (see main text) By contrast, for case "D" the matrix element for tunnelling between the energetically aligned LLs $n_{b}=3$ and $n_{t}=1$ is high.

\section{Conductance peaks for $V_{g}<0$}

We now analyse the features that appear in the $G\left(V_{b}, V_{g}\right)$ colour maps at low $\left|V_{b}\right| \lesssim 0.2 \mathrm{~V}$ for $V_{g}<0$ when $B$ $=2 \mathrm{~T}$ and $4 \mathrm{~T}$. These features occur whenever the chemical potential in either the bottom or top layer is aligned 

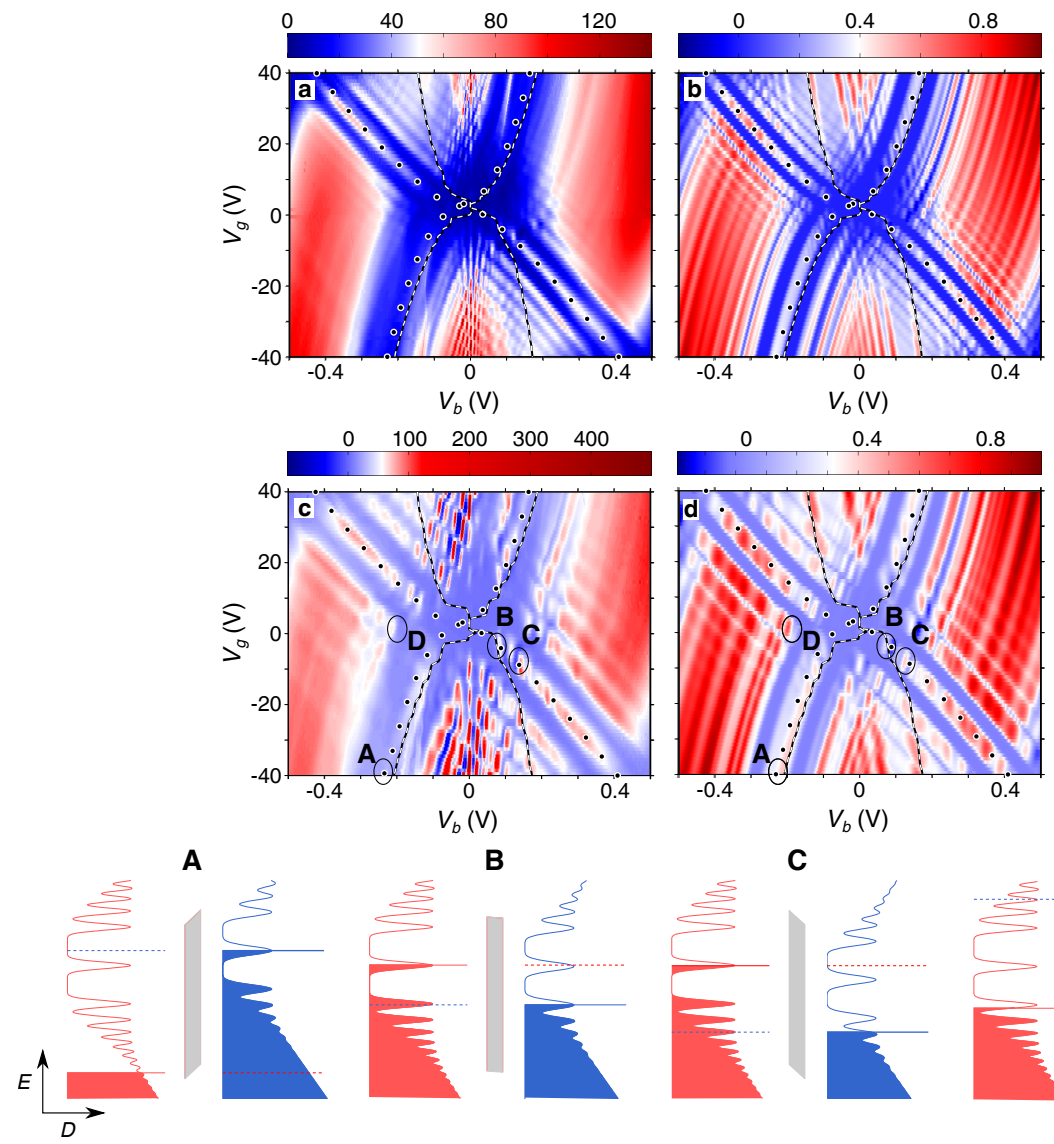

A

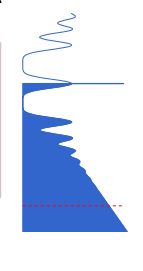

B

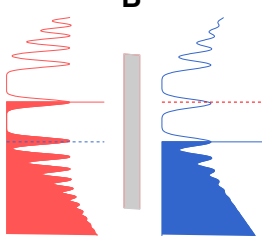

C

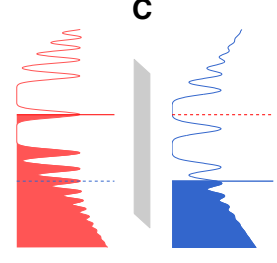

D

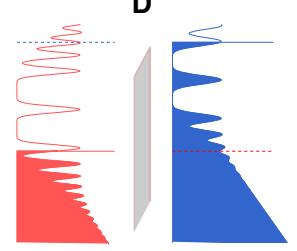

Figure 8: Colour maps showing $G\left(V_{b}, V_{g}\right)$ measured (a) and calculated (b) when $B=2 \mathrm{~T}$ and when $B=4 \mathrm{~T}$ (c measured, $\mathbf{d}$ calculated). Colour scales for $\mathbf{a}, \mathbf{c}(\mathbf{b}, \mathbf{d})$ are in $\mu \mathrm{S}$ (arbitrary units). Black and white dashed curves enclose islands within which only conduction-conduction $\left(V_{g}>0\right)$ or valence-valence $\left(V_{g}<0\right)$ tunnelling occurs. Filled black circles running top left to bottom right (bottom left to top right) show loci along which the chemical potential the bottom (top) layer intersects with the Dirac point in that layer. Lower panels A-D show the density of states, $D$, calculated versus energy, $E$, in the bottom (red) and top (blue) graphene electrodes and correspond to the features labelled A-D in colour maps c and d. Horizontal red and blue dashed lines show position of the chemical potentials in the bottom and top electrodes.

energetically with one of the LLs in the top or bottom layer respectively. The resulting local maxima in $G\left(V_{b}, V_{g}\right)$ occur at similar positions in the measured (Figs. 8a,c) and calculated (Figs. 8b,d) colour maps. However, when $B=2 \mathrm{~T}$, the theoretical results reveal many more features than the measured data. This is because our calculations assume a constant LL width and therefore omit the increased LL broadening that could occur at high $V_{b}$ in the actual device, e.g. due to electron heating. However, the general features of the measured and calculated colour maps are similar, in particular the positions of the resonant peaks and the width and shape of the X-shaped low $G$ region.

In Figs. 9a and $\mathbf{b}$ we show an enlargement of Fig. 8c and $\mathbf{d}$ focusing on the series of conductance peaks found for low $V_{b}$ and negative $V_{g}$ when $B=4 \mathrm{~T}$. To model the data at low $V_{b}$, where electron heating is low, we use a narrower broadening $\left(\Gamma_{b}=3 \mathrm{meV}\right.$ and $\left.\Gamma_{t}=5 \mathrm{meV}\right)$ than used for the full range of bias voltage. There is very good correspondence of the positions of the peaks in the modelled and measured data. As for the local conductance peaks considered previously, the peaks arise from a series of alignments of LLs of different index and the alignments of the chemical potentials. To aid understanding of these features we highlight two series of resonant peaks labelled, respectively, "A-F" and "i-v" showing the alignment of the LLs and the position of the chemical potentials in the graphene layers.

\section{MODEL FOR NON-CHIRAL ELECTRONS}

To understand the effect of pseudospin on our conductance calculations, in this section we derive a model for nonchiral electrons. This model has the same structure as that presented for chiral electrons except that the electron 


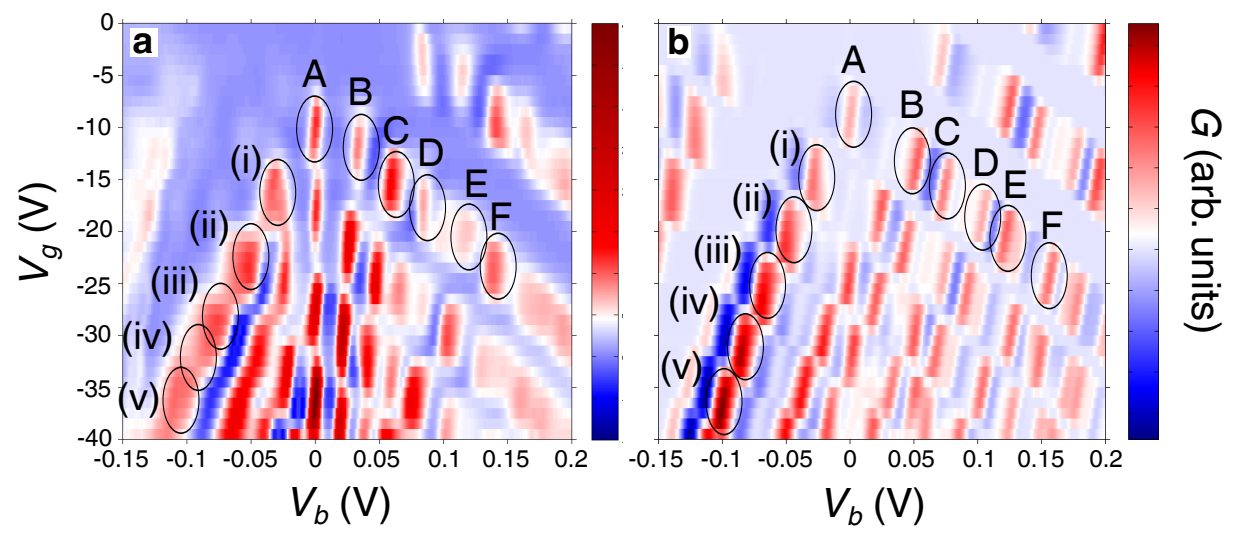

A



C



D

$\mathbf{E}$
$\Omega$

Figure 9: Colour maps showing comparison of a measured and $\mathbf{b}$ modelled $G\left(V_{g}, V_{b}\right)$ for $V_{g}<0$ and $V_{b}<0.2 \mathrm{~V}$ when $B=4 \mathrm{~T}$. Theory curves are calculated with $\Gamma_{b}=3 \mathrm{meV}$ and $\Gamma_{t}=5 \mathrm{meV}, \sigma_{s}=9 \mathrm{~nm}$, and misalignment angle $=1^{\circ}$. Circled features and corresponding inset plots show the alignments of LLs in bottom (red) and top (blue) electrodes with the chemical potentials indicated by the top of the block colour.

states are described by a single-component wavefunction of the form

$$
\Psi_{n_{b, t}, k_{y, b, t}}^{K^{+}}(\mathbf{r})=\frac{1}{\sqrt{L}} \exp \left(i k_{y, b, t} y\right) \phi_{\left|n_{b, t}\right|},
$$

where the variables have the same meaning as those given in Section. Although this form of the wavefunction does not correspond to a physical system, it is similar to LL states in III-V materials with massless Fermions and it allows us to distinguish clearly the effect of chirality on the measured and calculated conductance. In Fig. 10a we show $W\left(n_{b}, n_{t}\right)$ calculated for non-chiral $\mathbf{a}$ and chiral $\mathbf{b}$ electrons (see Fig. $3 \mathbf{c}$ of the main text). The figure reveals that for non-chiral electrons, a, transitions between equivalent ( $\mathrm{c}-\mathrm{c}$ and $\mathrm{v}-\mathrm{v}$ ) and different bands ( $\mathrm{v}-\mathrm{c}$ and $\mathrm{c}-\mathrm{v})$, have the same magnitude (i.e. the colour map is invariant under 90 degree rotations); by contrast, for chiral electrons, $\mathrm{c}-\mathrm{c}$ and $\mathrm{v}-\mathrm{v}$ transitions are strongly enhanced compared to $\mathrm{v}-\mathrm{c}$ and $\mathrm{c}-\mathrm{v}$ transitions (e.g. the rates are higher in the top right and lower left corners than they are in the top left and lower right corners).

Fig. 11 compares our conductance calculations for chiral electrons, see Section, with those for non-chiral electrons. When $B=2 \mathrm{~T}$ and $4 \mathrm{~T}$, within the upper (lower) region, above (below) the dashed curves, the measurements (Figs. $11 \mathbf{a}, \mathbf{d}$ ) and full chiral calculations (Figs. 11c,f) reveal that the conductance peak amplitudes are largest where c-c $(\mathrm{v}-\mathrm{v})$ transitions dominate (i.e. within the regions bounded by the dashed curves). Increasing or decreasing $V_{b}$ outside of these regions suppresses the conductance peaks. By contrast, in the calculations using non-chiral wavefunctions [Eq. (33)], the conductance peaks in the lower and upper regions have an almost constant amplitude over the whole range of $V_{b}$ (see Figs. 11b,e). This is because the matrix elements in the chiral calculations depend on the initial and final band of the tunnelling electron and is enhanced for transitions between equivalent bands compared to those between different bands. However, in our non-chiral model, the matrix elements are equal for transitions between states with the same LL index magnitudes, irrespective of whether those states are in equivalent or different bands, and, therefore, the conduction peak amplitudes are constant across the lower and upper regions bounded by the dashed curves in Fig. 11.

A changeover between regions of high and low conductance can also be seen in our recent studies of the $G\left(V_{b}, V_{g}\right)$ characteristics of similar tunnel structures when $B=0$ [8]. However, in the present work, the changeover is more pronounced because the quantising magnetic field strongly reduces the number of distinct tunnel-coupled states that contribute to the current flow; i.e. the effect of chirality is strongly magnetic field dependent. Consequently, the 

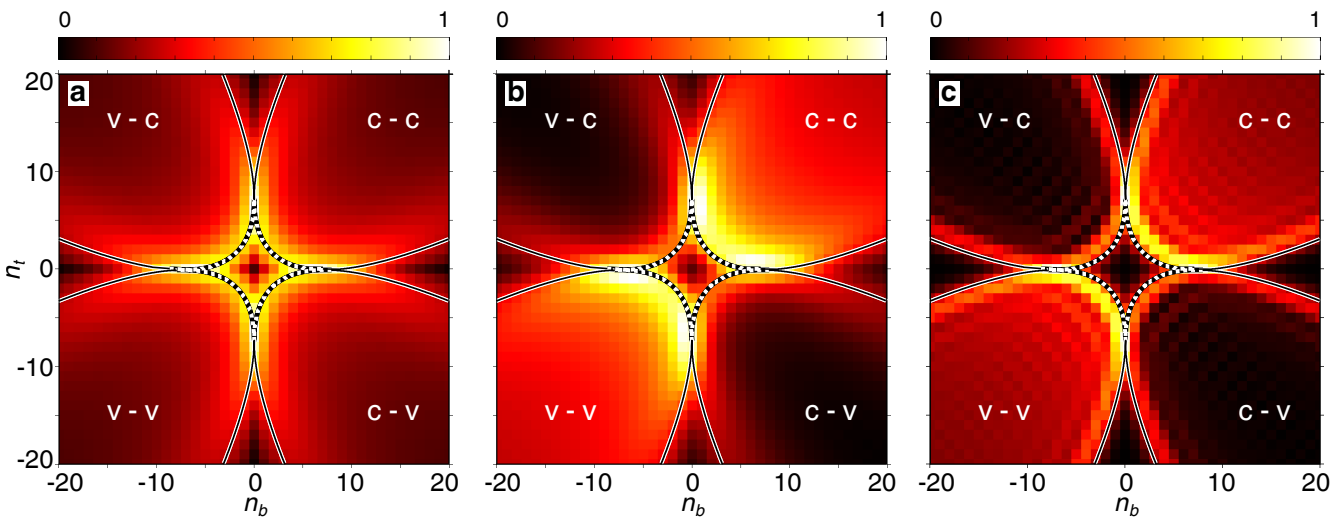

Figure 10: Colour maps showing normalised tunnelling rates, $W\left(n_{b}, n_{t}\right)$ [see Eq. (28)], between LLs with indices $n_{b}$ and $n_{t}$ in the bottom and top electrodes calculated using non chiral $\mathbf{a}$ and chiral $\mathbf{b}$, $\mathbf{c}$ wavefunctions. $\mathbf{a}, \mathbf{b}$ are calculated for scatteringassisted transitions (taking $\sigma_{s}=9 \mathrm{~nm}$ ) and $\mathbf{c}$ is calculated for momentum conserving transitions in which no scattering occurs (i.e. $\left.V_{S}(x, y)=1\right)$.

conductance is more sensitive to differences in the matrix elements for tunnelling between equivalent and different bands, which originate from the chiral nature of the wavefunctions.

\section{Model for non-chiral electrons in zero field}

In zero field we calculate the current for chiral electrons using the model presented in $[8,9]$. We describe non-chiral electrons by plane wave states with the form

$$
\Psi_{\mathbf{k}_{b, t}}^{K^{+}}(\mathbf{r})=\frac{1}{\sqrt{A}} \exp \left(i \mathbf{k}_{b, t} \cdot \mathbf{r}\right)
$$

where $\mathbf{k}_{b, t}=\left(k_{x}, k_{y}\right)$ are the wavevectors in the bottom and top electrodes. Therefore the matrix element can be found using Eq. 14:

$$
M_{b t}=\frac{\Xi}{A} \exp \left(-\sigma_{s}^{2}\left|\mathbf{k}_{\mathbf{b}}-\mathbf{k}_{\mathbf{t}}-\Delta \mathbf{K}^{ \pm}\right|^{2} / 2\right)
$$

We then calculate the current by using this form of the matrix element in Eq. (24) summing over $k$-states in zero field.

\section{THE EFFECT OF ELECTRON SCATTERING ON THE TUNNELLING RATES}

As explained above, our model includes the effect of elastic scattering by a potential landscape, $V_{S}(x, y)$, that originates from partially screened impurities near the graphene layers. The Fourier component of the tunnelling matrix element, $V(q)$, is largest when momentum is conserved, i.e. when $q=0$, so momentum conserving and nearmomentum conserving processes dominate. Even wavevector changes corresponding to the width of the scattering potential in $\mathrm{k}$-space $\left(q \sim 10^{8} \mathrm{~m}^{-1}\right)$ are smaller than the Dirac cone displacement $\Delta K \sim 3 \times 10^{8} \mathrm{~m}^{-1}$ due to the misorientation of the graphene layers. As discussed above, the Dirac cone displacement acts as an in-plane pseudomagnetic field and therefore changes the kinetic momentum during tunnelling transitions by effectively bending the electron orbits. In addition, the measured Landau level broadening of $8 \mathrm{meV}$ at $B=4 \mathrm{~T}$ corresponds to a $k$ range far smaller $\left(\sim 10^{7} \mathrm{~m}^{-1}\right)$ than that produced by the elastic scattering. Therefore the angular misorientation of the graphene layers has the largest influence on the tunnelling transitions and, hence, largely determines the shape of the current-voltage characteristics. Although the device is of high quality, a small amount of disorder is present and this gives rise to potential fluctuation which can scatter the electrons and broaden the resonances. The broadening of the resonant peak at $B=0$ is due to this, as discussed in Ref. [6, 8]. The scattering potential merely acts to broaden the features in the tunnelling rate map, as can be seen by comparing Fig. $3 \mathbf{b}$ (equivalent to Fig. 4c in the main text) and Fig. 3c above, which shows the tunnelling rates calculated without scattering. Consequently, the current-voltage 

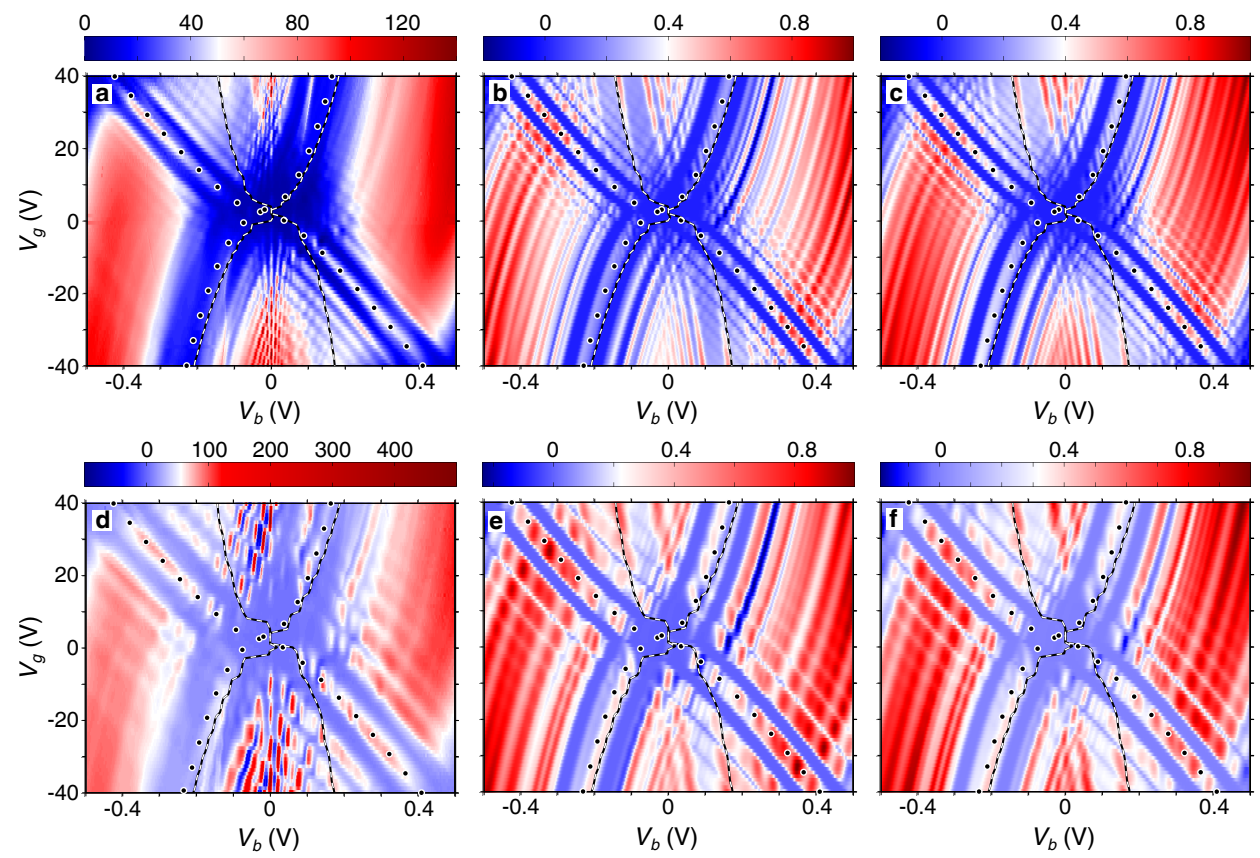

Figure 11: Colour maps showing $G\left(V_{b}, V_{g}\right)$ maps when $B=2 \mathrm{~T}(\mathbf{a}-\mathbf{c})$ and $B=4 \mathrm{~T}(\mathbf{d}-\mathbf{f})$ measured a,d and calculated for non-chiral (b,e) and chiral (c,f) electrons. Colour scales are in $\mu \mathrm{S}$ for panels $\mathbf{a}$ and $\mathbf{d}$ and in arbitrary units for panels $\mathbf{b}, \mathbf{c}, \mathbf{e}$ and f. Black and white dashed curves enclose islands within which only conduction-conduction $\left(V_{g}>0\right)$ or valence-valence $\left(V_{g}<0\right)$ tunnelling occurs. Filled black circles running top left to bottom right (bottom left to top right) show loci along which the chemical potential the bottom (top) layer intersects with the Dirac point in that layer.

curves is predominantly determined by the misalignment of the graphene electrodes. Our model accounts successfully for the measured broadening both of the main resonant peak at $B=0$ and of the inter-Landau level resonances in the $G\left(V_{b}, V_{g}\right)$ map.

\section{MAPPING CHIRALITY AND PSEUDO-SPIN ONTO SEMICLASSICAL ORBITS}

To gain insight into the effect of the two component form of the wavefunction and the associated chirality and pseduospin on the tunnelling matrix elements, we consider a semiclassical model in which the Lorentz force, $F_{l}=$ $-e(\mathbf{v} \times \mathbf{B})$, induced by the perpendicular magnetic field, causes the electrons to perform cyclotron orbits in real space and, since $\hbar \mathbf{k}=-e(\mathbf{r} \times \mathbf{B})$ also in $k$ space. The energy of the electron is quantised into LLs given by Eq. (6) and, thus, the radius in $k$-space of the orbit corresponding to the $\mathrm{n}^{t h}$ LL is given by $\kappa=\sqrt{2|n|} / l_{B}$. The electron therefore effectively performs cyclotron orbits around the surface of the Dirac cone (see Fig. $4 \mathbf{a}, \mathbf{b}$ of the main text). As the semiclassical electron performs cyclotron orbits in $k$-space it is interesting to note that its pseudospin direction, $\langle\sigma\rangle=s( \pm \cos \varphi, \sin \varphi)$ also rotates, where \pm refers to the two non equivalent valleys. The misalignment of the Dirac points in the two layers mean that orbit centres of the electrons in the two layers are similarly misaligned in $k$-space by $\boldsymbol{\Delta} \mathbf{K}$. To illustrate this, in Fig. 12 a we show the relative position of the $k$-space electron cyclotron orbits in the bottom (red) and top (blue) electrodes when $n_{b}=8$ and $n_{t}=10$.

In our semiclassical model the electron can make a momentum conserving transition between the two electrodes when the two orbits intersect at positions $\mathbf{k}_{\mathbf{1}}$ and $\mathbf{k}_{\mathbf{2}}$ (see labelled, filled black circles). In graphene, the two component nature of the electron wavefunction means that the transition probability depends on the positions of $\mathbf{k}_{1}$ and $\mathbf{k}_{2}$. To reveal this effect, we calculate the tunnelling probability at $\mathbf{k}_{1}$ and $\mathbf{k}_{2}$ for electrons in zero magnetic field. The Bloch electron wavefunctions, at the $K^{+}$point, when $B=0$ in the bottom and top electrodes have the form:

$$
\psi_{b, t}=\left(u_{b, t}^{\alpha}(\mathbf{r})+u_{b, t}^{\beta}(\mathbf{r}) s_{b, t} e^{\varphi^{b, t}}\right) e^{i \mathbf{k}_{b, t} \cdot \mathbf{r}}
$$

where $u_{b, t}^{\alpha, \beta}$ are the cell periodic parts of the Bloch functions, at the Dirac points in the bottom and top electrodes, corresponding to the two distinguishable atoms $\alpha$ and $\beta$ in the unit cell. The probability of tunnelling between the two electrodes at the two points of intersection between the semiclassical cyclotron orbits is proportional to the overlap 

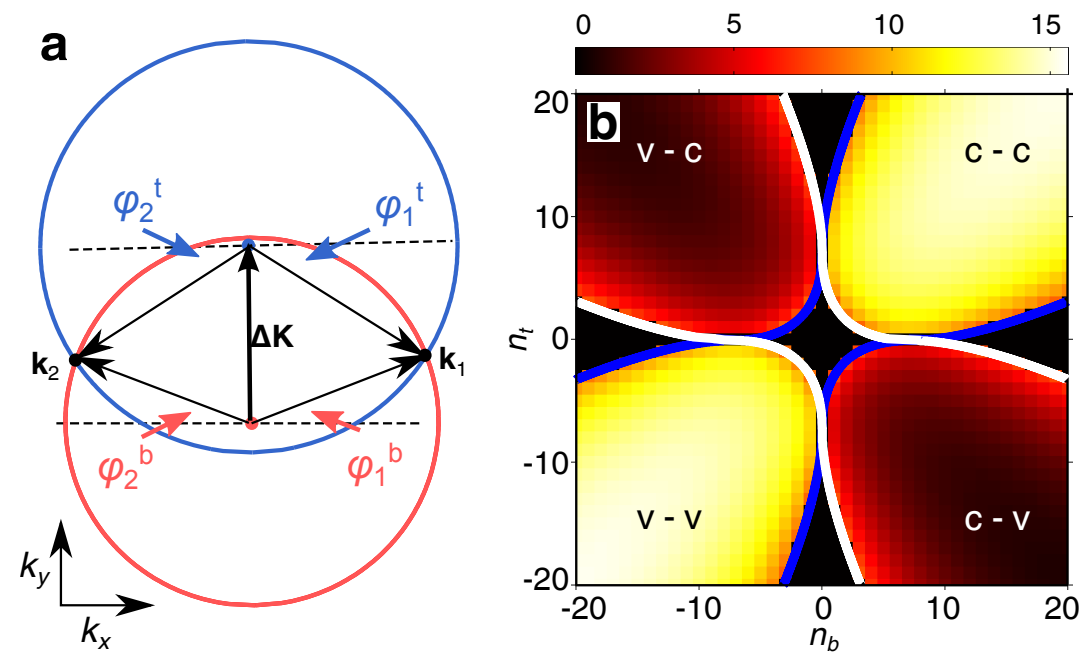

Figure 12: a $k$-space cyclotron orbits corresponding to the $n_{b}=8$ (red) and $n_{t}=10$ (blue) Landau states with orbit centres separated by $\Delta \mathbf{K}$, showing the two points of intersection between the orbits, $\mathbf{k}_{1}$ and $\mathbf{k}_{2}$ and the orientation of the wavevectors in the bottom $\left(\varphi_{1,2}^{b}\right)$ and top $\left(\varphi_{1,2}^{t}\right)$ graphene layers. b colour map of $W_{\text {chiral }}\left(n_{b}, n_{t}\right)$ [see Eq. (37)], blue loci show transitions where the electron pseudospin in the two layers have the same orientation and white loci where the pseudospin in the two layers is rotated by $180^{\circ}$ (see final section of the main text).

integrals of the wavefunctions in the two layers. Due to the small relative rotation of the two layers, the spatial overlap integrals of the cell-periodic parts of the wavefunctions $\int d S u^{* \alpha, \beta}(R(\theta) \mathbf{r}) u^{\alpha, \beta}(\mathbf{r})$ are approximately equivalent for all combinations of $\alpha$ and $\beta$. Therefore the semiclassical tunnelling rate between states with quantum numbers $n_{b}$ and $n_{t}$ is determined by the sum of the tunnelling probabilities at $\mathbf{k}_{1}$ and $\mathbf{k}_{2}$, i.e. is given by

$$
W_{\text {chiral }}\left(n_{b}, n_{t}\right) \propto\left|g\left(s_{b}, s_{t}, \varphi_{1}^{b}, \varphi_{1}^{t}\right)\right|^{2}+\mid g\left(s_{b}, s_{t}, \varphi_{2}^{b},\left.\varphi_{2}^{t}\right|^{2},\right.
$$

where

$$
g\left(s_{b}, s_{t}, \varphi^{b}, \varphi^{t}\right)=1+s_{b} e^{i \varphi^{b}}+s_{t} e^{-i \varphi^{t}}+s_{b} s_{t} e^{i\left(\varphi^{b}-\varphi^{t}\right)}
$$

and the angles $\varphi_{1,2}^{b, t}$ are defined in Fig. 12. We therefore find that $W_{\text {chiral }}$ depends both on the orientation and the band of the initial and final state of the tunnelling electron and, hence, on the pseudospin and chirality of the electron at the points of intersection between the two cyclotron orbits. Physically, this dependence originates from interference between the electron wavefunctions on the $\alpha$ and $\beta$ sublattices in the two graphene layers. In Fig. $12 \mathbf{b}$ we show color maps of $W_{\text {chiral }}$ calculated for a range of $n_{B}$ and $n_{T}$ when $B=4 \mathrm{~T}$. We find strong enhancement (yellow) of $W_{\text {chiral }}$ for tunnelling between equivalent bands (c-c and v-v) and suppression (black) of $W_{\text {chiral }}$ when tunnelling occurs between different bands ( $\mathrm{v}-\mathrm{c}$ and $\mathrm{c}-\mathrm{v}$ ). This semiclassical analysis is in good correspondence, i.e. shows enhancement of tunnelling transitions between the same bands and suppression of those between different bands, with the tunnelling rates calculated using the full quantum model, see Fig. 10. Note that our semiclassical analysis deviates from the full quantum model at high Landau level indices, because it does not include the spatial form of the wavefunctions.

Along the blue (white) locus in Fig. 12 b, the pseudospin vector of the electron in the bottom and top electrodes is unchanged (reversed) by the tunnelling process. Consequently, these loci depend on whether the transitions are between equivalent or different bands. When the pseudospin is unchanged (blue loci) the tunnelling process is analogous to Klein tunnelling in planar graphene (see main text). 
[1] Liu, Y., Bian, G., Miller, T. and Chiang, T.-C. Visualizing Electronic Chirality and Berry Phases in Graphene Systems Using Photoemission with Circularly Polarized Light. Phys. Rev. Lett. 107, 166803 (2011).

[2] Young, A. F. and Kim, P. Quantum interference and Klein tunnelling in graphene heterojunctions. Nat. Phys. 5, 222 - 226 (2009)

[3] Katsnelson, M. I., Novoselov, K. S. and Geim A. K. Chiral tunnelling and the Klein paradox in graphene. Nat. Phys. 2, 620-625 (2006)

[4] Geim, A. K. and Grigorieva, I. V. Van der Waals heterostructures. Nature 499, 419-425, (2013).

[5] Britnell, L. et al. Field-effect tunneling transistor based on vertical graphene heterostructures. Science 335, 947-950, (2012).

[6] Britnell, L. et al. Resonant tunnelling and negative differential conductance in graphene transistors. Nat. Commun. 4, 1794, (2013).

[7] Fallahazad, B. et al. Gate-Tunable Resonant Tunneling in Double Bilayer Graphene Heterostructures. Nano Lett. 15, 1 (2015).

[8] Mishchenko, A. et al. Twist-controlled resonant tunnelling in graphene / boron nitride / graphene heterostructures. Nature Nanotech. 9, 808813 (2014).

[9] Feenstra, R. M, Jena, D. and Gu, G. Single-particle tunneling in doped graphene-insulator-graphene junctions. J. Appl. Phys. 111, 043711 (2012).

[10] Zhao, P., Feenstra, R. M., Gu, G. and Jena, D. SymFET: A Proposed Symmetric Graphene Tunneling Field-Effect Transistor. IEEE Trans. Electron Devices 60, 951957 (2013).

[11] Vasko, F. T. Resonant and nondissipative tunneling in independently contacted graphene structures. Phys. Rev. B 87, $075424(2013)$

[12] Brey, L. Coherent tunneling and negative differential conductivity in a graphene/h-BN/graphene heterostructure. Phys. Rev. Appl. 2, 014003 (2014).

[13] Eisenstein, J. P., Pfeiffer, L. N. and West K. W. Coulomb barrier to tunneling between parallel two-dimensional electron systems. Phys. Rev. Lett 69, 3804 (1992).

[14] Leadbeater, M. L., Sheard, F. W. and Eaves, L. Inter-Landau-level transitions of resonantly tunnelling electrons in tilted magnetic fields. Semicond. Sci. Technol. 6, 1021 (1991).

[15] Lee, G. H. et al. Electron tunneling through atomically flat and ultrathin hexagonal boron nitride. Appl. Phys. Lett. 99, 243114 (2011).

[16] Lopes dos Santos, J. M. B., Peres, N. M. R. and Castro Neto, A. H. Graphene Bilayer with a Twist: Electronic Structure. Phys. Rev. Lett. 99, 256802 (2007).

[17] Mele, E. J. Commensuration and interlayer coherence in twisted bilayer graphene. Phys. Rev. B 81, 161405 (2010).

[18] Bistritzer, R. and MacDonald, A. H. Transport between twisted graphene layers. Phys. Rev. B 81, 245412 (2010).

[19] Bistritzer, R. and MacDonald, A. H. Moiré bands in twisted double-layer graphene. Proc. Natl. Acad. Sci. U. S. A. 108, 122337 (2011).

[20] Li, G., Luican, A. and Andrei, E. Y. Scanning Tunneling Spectroscopy of Graphene on Graphite. Phys. Rev. Lett., 102, 176804 (2009).

[21] Ponomarenko, L. A. et. al Density of States and Zero Landau Level Probed through Capacitance of Graphene. Phys. Rev. Lett. 105, 136801 (2010).

[22] Shon, N. and Ando, T. Quantum Transport in Two-Dimensional Graphite System. J. Phys. Soc. Jpn. 67, 2421 (1998).

[23] Zheng, Y. and Ando, T. Hall conductivity of a two-dimensional graphite system. Phys. Rev. B 65, 245420 (2002).

[24] Li, G., Luican-Mayer, A., Abanin, D., Levitov, L. and Andrei, E. Y. Evolution of Landau levels into edge states in graphene. Nat. Commun. 4, 1744 (2013).

[25] Luican-Mayer, A. et al. Screening Charged Impurities and Lifting the Orbital Degeneracy in Graphene by Populating Landau Levels. Phys. Rev. Lett. 112, 036804 (2013).

[26] Zhang, Y, Tan, Y. W., Stormer, H. L., and Kim P, Experimental observation of the quantum Hall effect and Berry's phase in graphene. Nature 438, 201-204 (2005).

[27] Fu, Y.-S. et. al Imaging the two-component nature of Dirac-Landau levels in the topological surface state of Bi2Se3. Nat. Phys. 10 815-819 (2014).

[28] Miller, D. L. et. al Real-space mapping of magnetically quantized graphene states. Nat. Phys. 6 811-817 (2010).

[29] Zhang, Y. et. al Landau-Level Splitting in Graphene in High Magnetic Fields. Phys. Rev. Lett., 96, 136806 (2006).

[30] Pratley, L. and Zülicke, U. Magnetotunneling spectroscopy of chiral two-dimensional electron systems. Phys. Rev. B 88, $245412(2013)$

[31] Pratley, L. and Zülicke, U. Valley filter from magneto-tunneling between single and bi-layer graphene. Appl. Phys. Lett. 104082401 (2014).

[32] Pershoguba, S. S., Abergel, D. S. L., Yakovenko, V. M., and Balatsky, A. V. Effects of a tilted magnetic field in a Dirac double layer. Phys. Rev. B 91085418 (2015)

[33] Drallos, P. J. and Wadehra, J. M. Exact evaluation and recursion relations of two-center harmonic oscillator matrix elements. J. Chem. Phys. 85, 6524 (1986). 